\title{
Self-administered Phyto-acupressure for sleep disorders: a pilot study
}

\begin{abstract}
Objectives: To determine whether sleep quality in patients with sleep disorders is improved by Phyto-acupressure.

Design: YSOM is a prospective, observational 28 days pilot study with pre- and postprocedural measures and a self-recorded diary. Sleep onset latency, wake-up quality, early starts, awakenings and overall sleep quality were measured by numerical analogue scales.

Settings/location: The procedure was carried out across a large metropolitan area.
\end{abstract}

Subjects: A total of 25 patients (mean age 37 years) with sleep disorders without severe comorbidities took part.

Procedures: A home-based phyto-acupressure procedure, three times a day for 28 days was carried out.

Outcome measures: efficacy, safety, acceptability, compliance with home program, before, at the end, and during the trial (diary).

Findings: All patients followed the protocol correctly. No adverse effects were reported; therefore the skin tolerance of the device was very good. The use enabled a significant increase in overall sleep $(+73 \%$ at the fourth week) and wake-up quality $(+102 \%$ at W4). Issues with getting to sleep, the occurrence of night-time waking, early morning waking, as well as restless sleep were also vastly improved.

Conclusions: The results of this observational pilot study suggest that Phyto-acupressure is an effective and safe procedure for adult patients with sleep disorders that merit thorough evaluation through a randomized controlled trial.

Keywords: acupressure, Phyto-acupressure, self-administered acupressure, sleep disorders, traditional Chinese medicine, patient satisfaction, complementary medicine, integrative medicine, herbal medicine
Volume II Issue 3 - 2018

\author{
Doan Y,' Stern C, ${ }^{2}$ Liu B,' Baumelou $A^{2}$ \\ 'Integrated Center of Traditional Chinese Medicine, GHU Pitié \\ Salpêtrière-Paris 6 Pierre et Marie Curie, France \\ ${ }^{2}$ Ythera, I I-I3 avenue de la division Leclerc, 94230 Cachan, \\ France
}

Correspondence: Pr. Baumelou Alain. Integrated Center of Traditional Chinese Medicine. GHU Pitié Salpêtrière-Charles Foix. Sorbonne Université, 47-83 boulevard de l'hôpital, 7565। Paris Cedex 13, France, Email alain.baumelou@aphp.fr

Received: May 31, 2018 | Published: June 12, 2018

\section{Background}

Twenty to thirty percent ${ }^{1}$ of the French population suffer with a sleep disorder. There are numerous disadvantages to existing drugbased solutions: dependence, difficulty waking up and poor quality of alertness during the day. Ylo SOM is a Phyto-acupressure product to be used externally, specifically for sleep disorders. Ylo SOM is available in the form of an acupressure pen containing an active plant concentrate (Angelica sinensis, Albizia julibrissin, Paeonia albiflora et Eleutherococcus senticosus). The aim of this study is to evaluate efficacy for sleep disorders, skin tolerance, as well as comfort of use of Ylo SOM.

\section{Materials and methods}

\section{The subjects}

This is an open-label, intra-individual pilot study on 25 volunteer subjects (mean age of 37 years), presenting with common sleep disorders with no comorbidities for at least four months. Of the subjects included, 96\% presented with night-time waking, $84 \%$ presented with early morning waking and restless sleep, and lastly $80 \%$ had difficulties falling sleep (sleep onset latency). The subjects did not receive any other sleep disorder treatment, and did not have any addictions, to either alcohol or drugs for example. The patients had to carefully follow the initial instructions given by the advising doctor.

\section{Study design}

The duration of the study was 28 days. The subjects needed to use Ylo SOM three times per day. There are three steps to Ylo SOM application: location of specific acupressure points, based on the sleep disorder; application of the active plant concentrate to these points with a roll-on applicator; activation of these points with acupressure using the stainless-steel tip of the pen. Location of the points could be carried out using the paper leaflet or via the smartphone app. The active plant concentrate contained root and flower extract of Albizia Julibrissin, Paeonia Albiflora, Eleutherococcus Senticosus and Albizia Julibrissin flower extract. Three different types of questionnaires were completed: the REC questionnaire, completed on the day of inclusion; the DD questionnaire, completed each day and the ACC questionnaire, completed at the end of the study. The study was conducted according to the following design (Figure 1).

\section{Evaluation of tolerance}

On D0, an evaluation of the condition of the skin was carried out by a doctor. On D28, a double evaluation of tolerance by the doctor and by the volunteers was performed. Any sign observed by the investigator or reported by the subjects was considered to be a potential adverse effect. This adverse effect needed to be attributable to the product. 


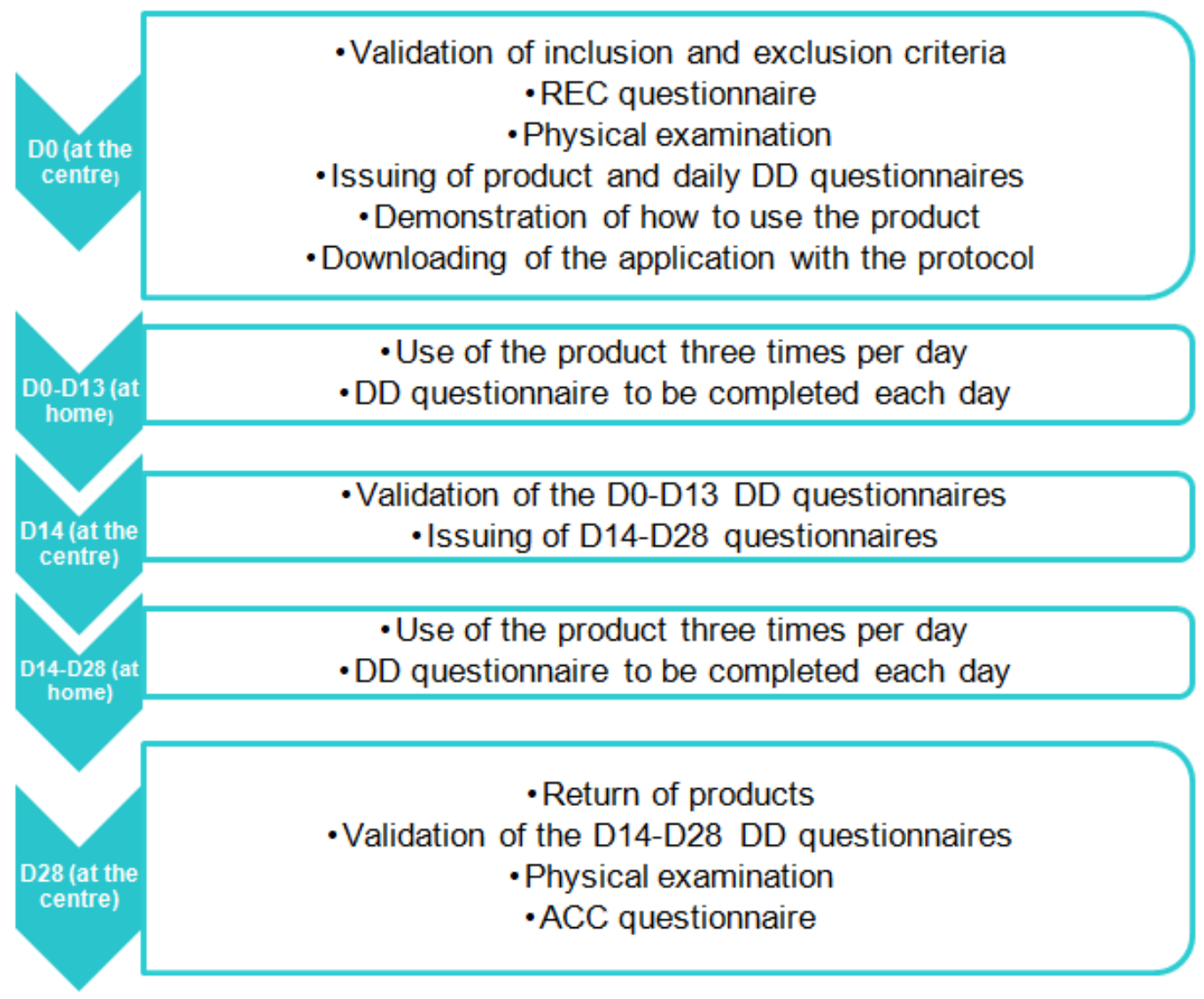

Figure I Study design regarding the tolerance, efficacy and comfort of use of Ylo SOM .

\section{Evaluation of efficacy on sleep quality, wake-up quality and sleep disorder symptoms}

The subjects evaluated various parameters: sleep quality, wake-up quality, difficulties in falling asleep, night-time waking, early morning waking and restless sleep using a numerical scale from 1 (very poor or no improvement) to 10 (excellent or very strong improvement). This evaluation was made at the time of recruitment on D0 using the REC questionnaire, then daily for 28 days using the DD questionnaire for overall sleep quality and overall waking quality. The results were compiled on a weekly basis: W1, W2, W3 and W4. The results show the mean score obtained on D0, W1, W2, W3 and W4 for overall sleep quality and overall wake-up quality and the mean score obtained on D1 (improvement seen following the first use of Ylo SOM), W1, W2, W3 and W4 for difficulties in falling asleep, night-time waking, early morning waking and restless sleep.

\section{Changes in emotional state of the patients}

Sleep quality may have an impact on emotional state and to a wider extent on quality of life. The subjects evaluated the following eight emotional states on D0 (REC questionnaire) and again on D28 (ACC questionnaire) using a numerical scale from 1 (not at all) to 10 (very high): stress, nervousness, anxiety, sadness, fatigue, enthusiasm, relaxation and calmness. The mean score was calculated for all subjects on D0 and D28.

\section{Evaluation of comfort, ease of use and convenience}

At the end of the study, the subjects recorded their level of satisfaction with regards to the comfort, ease of use and convenience of Ylo SOM.

\section{Statistical analyses}

Statistical analyses were carried out using IMB SPSS Statistic 22 software and the Wilcoxon test. Statistical significance was defined as $* \mathrm{p}<0.05, * * \mathrm{p}<0.01$ and $* * *$ if $\mathrm{p}<0.001$.

\section{Results}

Evaluation of the efficacy for sleep quality, wake-up quality and alertness

A significant increase in sleep quality of $+27 \%$ after one week, $+62 \%$ after 2 weeks and $+73 \%$ after 4 weeks was observed, as well as for wake-up quality, with $+52 \%$ at one week, $+88 \%$ after 2 weeks and $+102 \%$ after 4 weeks. Improvement in sleep and wake-up quality increased the longer Ylo SOM was used (Figure 2). Sleep quality and wake-up quality are improved after using Ylo SOM.

\section{Evaluation of the efficacy on sleep disorder symptoms}

A significant improvement in all symptoms was observed. This improvement gradually increased, the more the product is used (Figure 3). After two weeks' use, $100 \%$ of subjects felt there was an 
improvement; for $20.8 \%$ this was from the third day, for $33 \%$, from the first week and for $46 \%$, from the second week.

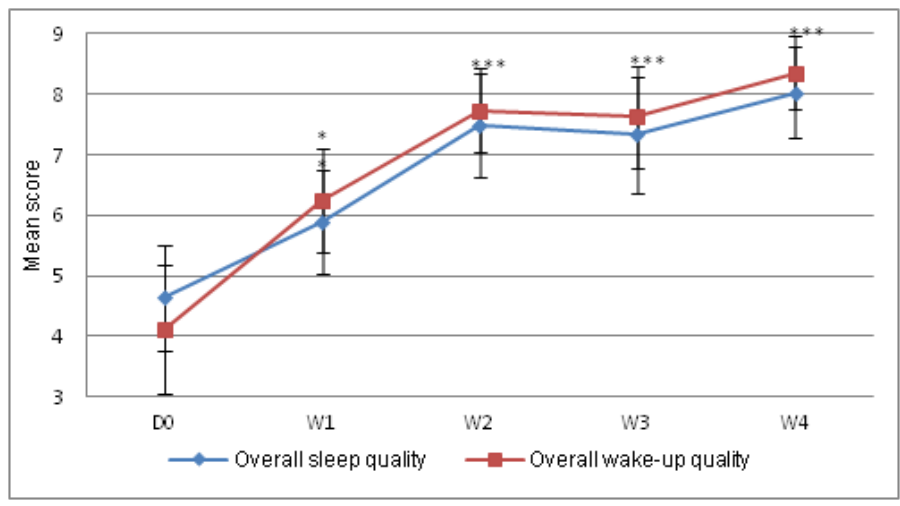

Figure 2 Changes in overall sleep and wake-up quality.

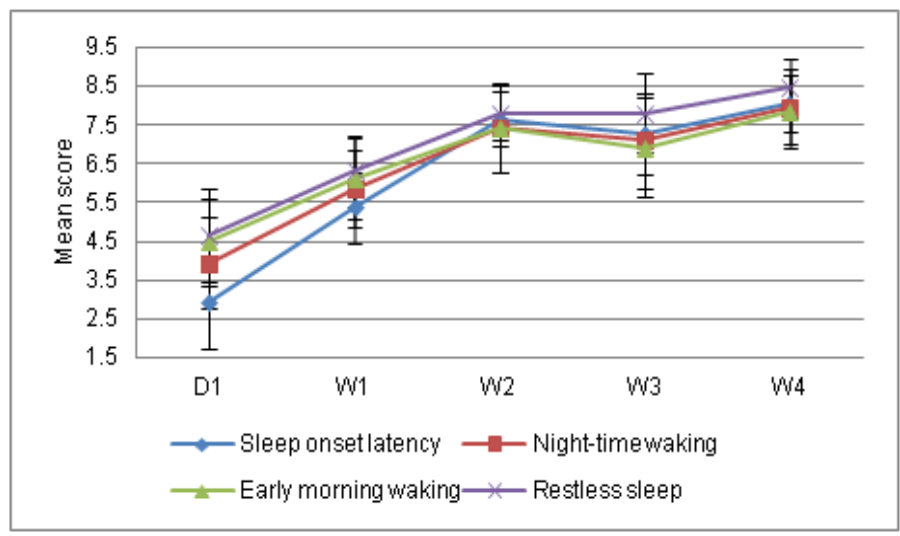

Figure 3 Changes in sleep disorder symptoms.

\section{Changes in emotional state of the patients}

The use of Ylo SOM over 28

days had a considerable impact on symptoms related to stress, with a $48 \%$ reduction. This was also seen for fatigue, which saw a $46 \%$ reduction. There was also a positive impact observed for nervousness, calmness and relaxation, as well as enthusiasm, whereas, for sadness and anxiety there were no significant variations (Figure 4).

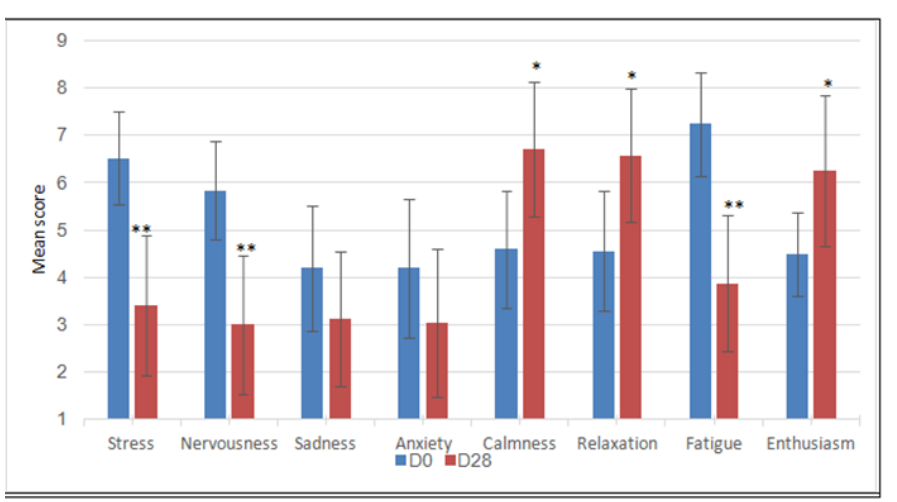

Figure 4 Evaluation of emotional state - Mean score.

\section{Evaluation of tolerance}

No adverse effects were reported, and therefore the skin tolerance of Ylo SOM was very good.

\section{Evaluation of comfort, ease of use and convenience}

Of the subjects included, $95.6 \%$ found Ylo SOM pleasant or very pleasant to use. Regarding ease of use, $83 \%$ of subjects found the protocol easy to understand and $87.5 \%$ found the duration of application acceptable. Locating the acupressure points was easy for $67 \%$ and moderately easy for $29 \%$.

\section{Discussion}

Acupressure is commonly used in some cultures and countries to manage symptoms. This technique is non-invasive and has proven to be a useful solution for a wide variety of symptomatic patients. ${ }^{2,3}$ We are reporting on a device that combines acupressure and the cutaneous application of plant extracts to acupressure points. There are some studies reporting a similar procedure. ${ }^{4,5}$ Phyto-acupressure or the application of a herbal preparation on acupressure points is first mentioned in the Wu Shi Er Bing Fang, "recipes for 52 ailments" and is still very popular in China, especially in the prevention and treatment of allergic rhinitis. ${ }^{6,7}$

We are reporting on an uncontrolled pilot study of phytoacupressure in patients with insomnia. The acupressure points used for this trial are listed in Appendix 1. Shenmen (HT7), the most widely used acupressure point in existing treatment protocols for sleep disorders, ${ }^{8}$ was used in all our protocols. Additional acupressure points were selected according to the predominant sleep disorder presented.

Appendix I: Ylo SOM acupressure points and their correspondence with international nomenclature

\begin{tabular}{|c|c|c|}
\hline & $\begin{array}{l}\text { European } \\
\text { nomenclature }\end{array}$ & $\begin{array}{l}\text { International } \\
\text { nomenclature }\end{array}$ \\
\hline \multirow{4}{*}{ Difficulty in falling asleep } & $2 \mathrm{EX}$ & $2 \mathrm{EX}$ \\
\hline & $7 C$ & $7 \mathrm{HT}$ \\
\hline & 20DM & $20 \mathrm{GV}$ or DU \\
\hline & I RN & I KI \\
\hline \multirow{4}{*}{ Night-time waking } & $2 \mathrm{EX}$ & $2 \mathrm{EX}$ \\
\hline & $7 C$ & $7 \mathrm{HT}$ \\
\hline & $2 \mathrm{Gl}$ & $2 \mathrm{LI}$ \\
\hline & $3 \mathrm{RN}$ & $3 \mathrm{KI}$ \\
\hline \multirow{4}{*}{ Restless sleep } & $2 \mathrm{EX}$ & $2 \mathrm{EX}$ \\
\hline & $7 C$ & $7 \mathrm{HT}$ \\
\hline & $6 \mathrm{MC}$ & $6 \mathrm{PC}$ or $\mathrm{HC}$ \\
\hline & $3 \mathrm{~F}$ & $3 \mathrm{LV}$ \\
\hline \multirow{4}{*}{ Early morning waking } & $2 \mathrm{EX}$ & $2 \mathrm{EX}$ \\
\hline & $7 C$ & $7 \mathrm{HT}$ \\
\hline & 6 RT & $6 \mathrm{SP}$ \\
\hline & $4 \mathrm{Gl}$ & $4 \mathrm{LI}$ \\
\hline \multirow{4}{*}{$\begin{array}{l}\text { Sleep disrupted by } \\
\text { digestion }\end{array}$} & $2 \mathrm{EX}$ & $2 \mathrm{EX}$ \\
\hline & $7 C$ & $7 \mathrm{HT}$ \\
\hline & 12 RM & $12 \mathrm{CV}$ \\
\hline & $25 \mathrm{E}$ & $25 \mathrm{ST}$ \\
\hline
\end{tabular}


Plants were selected based on their use in traditional Chinese medicine. The topical application of medicinal plant-based preparations to acupressure points currently uses a paste or cataplasm to treat different diseases in a wide range of Chinese clinical departments. The most common indications are for asthma, cough, abdominal pain, facial paralysis and constipation in internal medicine departments, for cough, asthma and diarrhoea in paediatric departments, for lumbarsciatic pain in surgical departments, for rhino-sinusitis in ENT departments and for dysmenorrhea in gynaecology departments. ${ }^{9}$ The main plants used in Ylo SOM were selected for their properties and their affinity with the meridians.

The Ylo SOM device decreases early morning waking, nighttime waking, sleep onset latency and self-perceived sleep quality. High compliance toward acupressure was observed. No localised side effects at the acupressure points, general side effects or negative feelings were reported during the four weeks of everyday use.

The biomedical model for acupressure is not yet fully established. It is thought that the mechanism of action involves direct stimulation of the interstitial fluid and connective tissue, ${ }^{10}$ functional peripheral and central nervous system changes, plus changes in the circulation of cytokines and neurotransmitters. The effects of acupressure on sleep quality may be attributed to the activation of the parasympathetic nervous system, ${ }^{11}$ an increase in autonomous response and a reduction in psychological stress (8). A reduction in stress was also observed in our study.

The extent of the effect is disproportionally high compared with the usual placebo effect observed in controlled trials. Other factors that may be deemed to be relevant include the information provided and discussed prior to inclusion, the use of a smart phone app during the trial $^{12,13}$ and self-administration of treatment. ${ }^{14}$ These methodological bias may have affected the evaluation of the intrinsic efficacy of the device, however the overall assessment of the procedure represents true-life conditions and the actual effect on patient satisfaction. This trial provides several indications suggesting that this device has a potential to improve self-perceived sleep quality without any adverse effects. The procedure is easy to learn, non-invasive, and can be selfadministered. Future controlled studies are required to investigate the inherent efficacy of the device and to monitor the effectiveness in minority populations with insomnia: children, pregnant women, chronic renal failure patients, residents in long-term care facilities.

\section{Conclusion}

The results of this observational pilot study suggest that Phytoacupressure is an effective and safe procedure for adult patients with sleep disorders that merit thorough evaluation through a randomized controlled trial.

\section{Acknowledgments}

None.

\section{Conflict of interest}

The research project was partially sponsored by Lutech Society for the Acceleration of Technology Transfer (SATT Lutech) for GHU Pitié Salpêtrière-Paris 6 Pierre et Marie Curie.

\section{References}

1. Léger D, Bayon V. Societal costs of insomnia. Sleep Med Rev. 2010;14(6):379-389.

2. Lee EJ, Frazier S. The efficacy of acupressure for symptom management: a systematic review. J Pain Symptom Manage. 2011;42(4):589-603.

3. Song HJ, Seo HJ, Lee H, et al. Effect of self-acupressure for symptom management: a systematic review. Complement Ther Med. 2015;23(1):68-78.

4. Fung JKK, Tsang HW. Management of behavioural and psychological symptoms of dementia by an aroma-massage with acupressure treatment protocol: a randomized clinical trial. J Clin Nurs. 2017;27(9-10):18121825 .

5. Yang MH, Lin LC, Wu SC, et al. Comparison of the efficacy of aromaacupressure and aromatherapy for the treatment of dementia associated agitation. BMC Complement Altern Med. 2015;15:93.

6. Cai-Yu-Zhu W, Ya-Fei L, Li Z, et al. A systematic and narrative review of acupuncture point application therapies in the treatment of allergic rhinitis and asthma during dog days. Evidence-Based Complementary and Alternativ Medicine. 2015;10.1155/2015/846851.

7. Tai CJ, Chang CP, Huang CY, et al. Efficacy of Sanfujiu to treat allergies: patient outcomes at 1 year of treatment. Evidence-Based Complementary and Alternative Medicine. 2007;4(1):241-246.

8. Waits A, Tang YR, Cheng HM, et al. Acupressure effect on sleep quality: A systematic review and meta-analysis. Sleep Med Rev. 2016;37:24-34.

9. Zhang X, Zhang XP, Jia CS, et al. Basic rules and characteristics of acupoint application therapy based upon data mining. Zhen Ci Yan Jiu. 2012;37(5):416-421

10. Chen CW, Tai CJ, Choy CS, et al. Wave-induced flow in meridians demonstrated using photoluminescent bioceramic material on acupuncture points. Evid Based Complement Altern Med. 2013;2013:739293.

11. Selfridge N. Acupressure: the evidence presses on. Integr Med Alert. 2012;15:64-67.

12. Takeuchi S, Horluchi S. Randomized controlled trial using smartphone website vs leaflet to support antenatal perineal massage practice for pregnant women. Women Birth. 2016;29(5):430-435.

13. Horsch CH, Lancee J, Griffioen-Both F, et al. Mobile Phone-Delivered Cognitive Behavioral Therapy for Insomnia: A Randomized Waitlist Controlled Trial. J Med Internet Res. 2017;19(4):e70.

14. Crawford C, Lee C, Freilich D. Active Self-Care Therapies for Pain (PACT) Working Group: Effectiveness of active self-care complementary and integrative medicine therapies: options for the management of chronic pain symptoms. Pain Med. 2014; 15(Suppl 1):S86-95. 